\title{
Wenn Folter kein Asylgrund mehr ist
}

Hüssein fällt es schwer, über seine Vergangenheit zu sprechen. Aus politischen Gründen war der Zahnarzt in Syrien brutal gefoltert worden. Spezialärztliche Zeugnisse belegen sowohl physische wie psychische Spuren der Misshandlung. Rund 26000 anerkannte Flüchtlinge leben heute in der Schweiz. Sie waren Opfer schwerer Verfolgung oder davon bedroht. Geht es nach der Asylinitiative der SVP, könnten Flüchtlinge in der Schweiz bald kein Asyl mehr erhalten: Wer über einen «sicheren Drittstaat» einreist, soll sofort dorthin weggewiesen werden. Weil die Nachbarländer der Schweiz als sicher gelten dürfen und bis zu 98 Prozent aller Asylsuchenden auf dem Landweg fliehen, würden praktisch alle ausgeschlossen: Gefolterte, Verfolgte, Traumatisierte, Bürgerkriegsflüchtlinge. Tatsächlich dürfte die Wegweisung in die Nachbarstaaten nicht ohne weiteres gelingen. Voraussetzung ist nämlich, dass der frühere Aufenthalt belegt werden kann. Scheitert die Rückübernahme, muss die Schweiz die Gefährdung im Heimatland abklären. Selbst wenn aber zweifelsfrei feststeht, dass dort Verfolgung, Inhaftierung und Folter drohen, bliebe die Asylgewährung künftig ausgeschlossen. Die Konsequenzen sind drastisch: Gefährdete Kinder und Ehegatten müssten im Heimatland bleiben und könnten nicht in Sicherheit gebracht werden. Die Integration von Flüchtlingen würde verhindert statt gefördert. Unter dem irreführenden Titel der Missbrauchsbekämpfung verlangt die Initiative nichts anderes als das Ende der humanitären Tradition der Schweiz.
Doch damit nicht genug: Die Initianten wollen die medizinische Behandlung auf Notfälle einschränken. In bestimmten Fällen wird Behandlung nur gewährt, wenn Betroffene mit den Behörden kooperieren. Wer wird die Notwendigkeit der Pflege beurteilen müssen und die Behandlung ablehnen? Wird Gesundheit zu einem Gut, das man sich durch Wohlverhalten erwerben muss? Und falls diese Forderungen Schule machen: Welche Personen ausserhalb des Asylbereichs würden als nächstes mit ähnlichen Sanktionen rechnen müssen? Bereits heute haben Asylsuchende als einzige Personengruppe keine freie Arztwahl.

Die Fürsorge soll auf blosse Unterkunft und Nahrung beschränkt werden. Doch Asylsuchende erhalten bereits heute 20 bis 50 Prozent tiefere Sozialleistungen als die übrige Bevölkerung, weitere Einschränkungen sind kaum möglich. Weil auch neue Arbeitsverbote vorgesehen sind, wird der Bedarf an staatlicher Fürsorge steigen. Die voraussehbare Zuspitzung sozialer Probleme und die Kostenexplosion wird dem Ruf nach neuen Verschärfungen Vorschub leisten. Die Initiative fördert die Probleme, die sie zu lösen vorgibt, ein Teufelskreis.

Bundesrat, Parteien und Hilfswerke engagieren sich gegen die Initiative. Am 24. November ist Abstimmung.

Jürg Schertenleib, Leiter Rechtsdienst, Schweizerische Flüchtlingshilfe SFH

Die Schweizerische Flüchtlingshilfe SFH ist die Dach- und Fachorganisation im Flüchtlingsbereich und setzt sich für den Schutz von Flüchtlingen ein. Regelmässig veröffentlicht sie Berichte über die Situation in den Herkunftsländern von Asylsuchenden und beschreibt dabei auch die medizinischen Behandlungsmöglichkeiten.

Informationen über die SFH unter www.fluechtlingshilfe.ch, zur Asylinitiative unter www.asylinitiative.ch. 